\title{
Portfolio Optimization under Cardinality Constraints: A Comparative Study
}

\section{Henri Claver Jimbo ${ }^{1 *}$, Isidore Seraphin Ngongo ${ }^{2,3}$, Nicolas Gabriel Andjiga ${ }^{3}$, Takeru Suzuki ${ }^{1}$, Charles Awona Onana ${ }^{3}$}

\footnotetext{
${ }^{1}$ Department of Applied Mathematics and Statistics, Laboratory of Big Data and Stochasticity, Waseda University, Tokyo, Japan ${ }^{2}$ Department of Applied Mathematics, Laboratory SAMM (Statistics Analysis and Multidisciplinary Modeling), University of Paris 1, Pantheon Sorbonne, Paris, France

${ }^{3}$ Department of Mathematics, Faculty of Science, University of Yaounde 1-ENS-Higher Training College, Yaounde, Cameroon Email: *jimbo_maths@yahoo.com
}

How to cite this paper: Jimbo, H.C. Ngongo, I.S., Andjiga, N.G., Suzuki, T. and Onana, C.A. (2017) Portfolio Optimization under Cardinality Constraints: A Comparative Study. Open Journal of Statistics, 7, 731-742.

https://doi.org/10.4236/ojs.2017.74051

Received: April 15, 2017

Accepted: August 28, 2017

Published: August 31, 2017

Copyright (C) 2017 by authors and Scientific Research Publishing Inc. This work is licensed under the Creative Commons Attribution International License (CC BY 4.0).

http://creativecommons.org/licenses/by/4.0/

\begin{abstract}
The Cardinality Constraint-Based Optimization problem is investigated in this note. In portfolio optimization problem, the cardinality constraint allows one to invest in $K \leq N$ assets out of a universe of $N$ assets for a prespecified value of $K$. It is generally agreed that choosing a "small" value of $K$ forces the implementation of diversification in small portfolios. However, the question of how small must be $K$ has remained unanswered. In the present work, using a comparative approach we show computationally that optimal portfolio selection with a relatively small or large number of assets, $K$, may produce similar results with differentiated reliabilities.
\end{abstract}

\section{Keywords}

Cardinality Constraints, Diversity, Portfolio Selection, Portfolio Reliability, Parametric Statistics

\section{Introduction}

Although the portfolio optimization problem has been studied using various analytical and numerical techniques for more than half a century, recent development of computer based methods has opened new horizon to research in computation finance. The problem of portfolio optimization has been rendered to be complex for direct solving by traditional numerical approaches when constraints that model investors sentiments and frictions are included in the mathematical model. An optimal stock portfolio investment strategy should show the investor how much to invest in each asset in a given portfolio. The 
decision variable of stock portfolio optimization is the weight of the asset in the portfolio. Once an optimal weight is obtained, the expected return and risk can be easily calculated. The solution to the stock portfolio optimization problem now lies in graphically obtaining the efficient frontier, which is a risk-return trade-off curve. Each point on the efficient frontier gives the minimum level of risk to take for an expected return or, alternatively, the maximum return one can expect for a given level of risk. Hence a rational investor would usually choose portfolios that occur on the efficient frontier, since these represent "optimal" portfolios.

The Markowitz approach for the solution of the Portfolio Selection Problem assumed a perfect market, ignoring transaction costs, taxes and permitting trading of securities at any proportions. Under these assumptions, the mathematical model is reduced to a quadratic optimization which can be directly solved by classical numerical methods [1] [2]. However, in practice, the portfolio managers operate under stricter constraints. We consider here, as in [3] [4] and [5], the basic constraints and, in addition, non-universal constraints. Under basic constraints, the weight allocated to each asset lies between zero and one, and the total of all weights sums to one, indicating a full investment. In practice, it is often the case that an investor chooses to invest a definite proportion of weights bounded by a range in specific stock and/or chooses to invest a proportion of weights in stocks related to specific sectors such as bank, energy, technology and so on, with sum total weights in each specific sector bounded by a limit. In the former case, the constraints are referred to as bounding constraints and in the latter case as class constraints.

We finally introduce the Conditional Value-at-Risk (CVaR) into the constraints and define the cardinality constraints to allow the investors to invest partially in smaller portfolios. The Cardinality Constraint, which is our main focus in this work, is adopted when the investor can only invest in $\mathrm{K}$ assets out of the universe of $\mathrm{N}$ assets, for a prespecified value of $\mathrm{K}$. Choosing a small value of $\mathrm{K}$ forces the implementation of diversification in small portfolios. A cardinality constrained optimization portfolio may then be viewed as a significant research topic in computational finance since the inclusion of such constraints turns the problem mathematically speaking in a mixed integer quadratic programming problem rendering it to be complex for direct solving by numerical methods. However, in some complex cases, a Genetic Algorithm (GA) approach may be required.

In this paper, we discuss the solution of complex cardinality constraint-based optimization problem but we also include all basic constraints (as part of the general constraints on portfolios over time), and implement a GA approach for investigating the solution of such a problem [1]. The GA is a member of the class of population-based stochastic search algorithms which are population-based and are developed from the ideas and principles of natural evolution. Our work is organized as follows: in Section two, we present the preliminary setup of the portfolio optimization problem; in Section three, we formulate the cardinality 
constraint-based optimization. In Section four we display and comparatively analyse results for the values $K=3,4,7$ and 8 . We discuss our results in Section five and end this work with a short conclusion in Section six.

\section{Overview of the Portfolio Problem}

We present in this section the mathematical foundation, propositions and theorems, and previous analytical results which support and validate our computational approach.

\subsection{Preliminary Setup}

This subsection presents an overview of the portfolio problem with respect to previous references [6]-[12]. The aim of this paper is to focus more precisely on solving the core optimization problem with differentiated size of assets and introduce the reader to new insights provided by our method, the ultimate goal being to let the data speak for themselves as much as possible. In our setup we hold $N$ assets $x_{1}, x_{2}, \cdots, x_{N}$ at time $t$ and wish to construct an optimal portfolio over given investment time periods. Our portfolio consists of $N$ traded assets numbered $1,2, \cdots, N$ held over a period of time, with $R_{j}$ the return on asset $j$ assumed to be a random variable. Assume $x_{j}(t)$ is the proportion of asset $j$ traded out of the total of all assets, and let $R_{1}, R_{2}, \cdots, R_{N}$ be the return rates of assets $1,2, \cdots, N$. We assume that $E\left[R_{j}\right]<\infty$ for all $j=1,2, \cdots, N$. Our aim is to invest our capital in $K \leq N$ assets in order to obtain some desirable characteristics of return rate on the investment. Denoting by $w_{1}, w_{2}, \cdots, w_{N}$ the fractions of the initial capital invested in assets, we have

$R(x)=R_{1} x_{1}+R_{2} x_{2}+\cdots+R_{N} x_{N}$ and the set of possible asset allocations may be defined as

$$
X=\left\{x \in R^{N}, x_{1}+x_{2}+\cdots+x_{N}=1 ; x_{j} \geq 0, j=1,2, \cdots, N\right\}
$$

In some applications one may introduce short selling; that is, allowing some $x_{j}$ to be negative. Other restrictions may limit the exposure to particular assets in given groups, by imposing restrictions on upper bounds of the $x_{j}$ s or their particular subset sum. One can also limit the absolute differences between the proportion $x_{j}(t)$ of our assets and some reference proportion $\overline{x_{j}}$ (which may represent the existing benchmark portfolio). One of the main difficulties of the Portfolio Optimization Problem remains the type of constraint being applied to the problem. In the next subsection we will present the concept of a dominance constrained portfolio and show how it simplifies the portfolio problem to some extent.

\subsection{Dominance Constrained Portfolio}

We begin by assuming that the benchmark random return rate $Y$ having a finite expected value is available. It may have the form $Y=R(\bar{x})$ for some benchmark portfolio $\bar{x}$, which may be some expected average of our current 
portfolio (see [7] [8] for details). Our intention is to have a return rate, $R(x)$, of the new portfolio preferable (in some sense) over Y: therefore we introduce the following optimization problem:

Find $\min f$

Subject to $R(x) \geq_{2} Y, x \in X$

Here $\geq_{2}$ denotes dominance and $f: X \rightarrow R$ is a concave continuous function. In particular, we may use $f(x)=E(R(x))$. Now we present some standard theorems and propositions which shall clarify our concepts.

Theorem 1. Let $P$ be a randomly constructed portfolio and $P_{1}$ the benchmark portfolio. The second order stochastic dominance constraint is equivalent to the continuum of $V a R$ constraints on portfolios. That is, $\operatorname{CVaR}_{\alpha}(P) \geq C V a R_{\alpha}\left(P_{1}\right)$ for all $\alpha \in(0,1]$, where $\mathrm{CVaR}_{\alpha}$ is the conditional Value-at-Risk at level $\alpha$.

Proof (by analogy to [9]). Let $P$ be any randomly constructed portfolio defined as a function of its assets. Consider a function $h$ that obeys the inequality $h(\alpha, P) \geq \operatorname{CVaR}_{\alpha}(P), \alpha \in(0,1]$. For $\alpha=0$, we get $h(0, P)=0$ (for details see [7] [13] and [14]).

The function $h(\alpha, P)$ is a curve defined on the portfolio $\mathrm{P}$ such that

$$
h(\alpha, P(x)) \geq \sup _{\kappa}\left\{\alpha \kappa-E\left[(\kappa-P(x))_{+}\right]\right\}=\sup _{\kappa}\{\alpha \kappa-g(P(x) ; \kappa)\}
$$

Observe that $h(\bullet, P(x))$ is the conjugate of the function $\left(P_{0}(x) ; n\right)$ such that $g(P(x) ; \kappa) \leq g\left(P_{0}(x)\right)$ for all $\kappa \in R$ (also notice that $g$ and $h$ are dual functions by the definition). This implies that $h(\alpha, P(x)) \geq h\left(\alpha, P_{0}(x)\right)$, but, since $g(P(x))$ is continuous by duality of the conjugate, we conclude that the initial capital allowed for risk exposure at level $\alpha \neq 0$ is given by the benchmark outcome $P_{1}$, and finally $-C_{V a R_{\alpha}}\left(P_{1}\right)=T_{\alpha}$, where $T_{\alpha}$ is the tail conditional expectation equivalent or the expected shortfall at fixed level $\alpha$.

Remark 1. If the portfolio is discrete, the stochastic dominance constraint can be replaced by infinitely many inequalities.

Proposition 1. Assume that the portfolio $P_{1}(x)$ defined as a linear function on assets has a discrete distribution with realisation $\left\{p_{i}\right\}, i=1, \cdots, N$. Then, the following equality holds.

$$
E\left[\left(p_{i}-P(x)\right)_{+}\right] \leq E\left[\left(p_{i}-P_{1}(x)\right)_{+}\right]
$$

where $\left(p_{i}-P(x)\right)_{+} \equiv \max \left(0, p_{i}-P(x)\right)$, recalling that $P$ denotes our portfolio.

Remark 2. The above proposition does not imply that the continuum of $C V a R$ constraints can be replaced by finitely many constraints of the form $\operatorname{CVaR}_{\alpha}(P(x)) \geq \operatorname{CVaR}\left(P_{1}(x)\right)$.

Proposition 2. Let $P=P(x)$ be the payoff or value of a portfolio at some future time and $0 \leq \alpha \leq 1$. If the underlying distribution of the portfolio $P$ is a continuous distribution then the Expected Shortfall $E S_{\alpha}$ is reduced to the Tail Conditional Expectation $T C E_{\alpha}$ 


$$
T C E_{\alpha}(P)=E S_{\alpha}(P)=E\left[-P \mid P \leq-\operatorname{VaR}_{\alpha}(P)\right]
$$

Let us now focus on extreme values of the portfolio. Basically a portfolio may have heavy tail distribution partly due to violent market movements. These large market movements, far from being accepted as simple outliers, focus the attention of all investor or players, since their magnitude may be such that they compose a fraction of the portfolio return aggregate over long period of time. These observations have motivated numerous theoretical and computational efforts to understand the intermittent role of various assets in the portfolio and model adequately the tail of distribution of their returns. Such studies are relevant for risk management purposes and also necessary for calculation of average risk (of loss in portfolios) which may be required to determine regulatory capital requirements [13] [14] [15] [16] and [17]. Now, given a series of nonoverlapping returns $x(t, \Delta t), t=0, \Delta t, 2 \Delta t, \cdots, n \Delta t$, the extremal (minimal and minimal) returns are defined as

$$
\begin{gathered}
m_{N}(x, \Delta t)=\min \{x(t+k \Delta t) ; k=1, \cdots, N\} \\
M_{N}(x, \Delta t)=\max \{x(t+k \Delta t) ; k=1, \cdots, N\}
\end{gathered}
$$

In terms of risk management $m_{n}(x, \Delta t)$ represents the worst relative loss over time horizon $\Delta t$ of an investor holding a portfolio $P(x(t))$. The question of how the properties of returns affect the probability distribution of $m_{n}(x, \Delta t)$ and $M_{n}(x, \Delta t)$ simultaneously over time seems to be quite attractive. Hence, if we knew the stochastic process generating the returns, we could easily evaluate the distribution of the extremes, but this is unfortunately not the case and that is where extreme value theory is needed. However we will not discuss the extreme value theory in this paper.

\subsection{Portfolio Reliability (Predicted and Realised Risk on a Portfolio)}

The reliability of a portfolio $P$ is a quantity that serves as a good measure to compare the goodness of the fit in the portfolio. For a given level of expected return, with $\sigma_{\text {pred }}$ and $\sigma_{\text {real }}$ to be the corresponding predicted and realised risks, the reliability $\mathfrak{R}$ of a portfolio is given by

$$
\mathfrak{R}=\left|\frac{\sigma_{\text {pred }}-\sigma_{\text {real }}}{\sigma_{\text {real }}}\right|
$$

A portfolio is more reliable when $\mathfrak{R}$ is small. In our setup the predicted risk is computed by our GA, whereas the realised risk uses the usual formula of variance. Here we use only a positive definite reliability to simplify the comparison between optimal reliability gains over generation time. In the next section we shall formulate the cardinality constraints based optimization problem.

\section{Cardinality Constraint-Based Optimization Problem}

To bring the element of time into play, we now expand our approach from vectors to matrices. Let $\mathrm{N}$ be the number of assets in the universe, $\mu_{i}=E\left(R_{j}\right)$ 
the expected return of the asset $i$, and $\sigma_{i j}$ the covariance between the returns of assets $i$ and $j$ in the historical data. Let $w=\left(w_{i j}\right)$ denote the weight matrix with elements representing the proportion of capital to be invested in asset $j$ at time $t=i$, and $K_{i}$ be the number of assets in which the investor decides to invest their capital at time $i$. Let $w_{j}$ denote the vector given by column $j$ of $w$ (that is, the weights over all time intervals for asset $j$, where $i$ denotes the ith entry of $\left.w_{j}\right), x_{0}$ the $N \times N$ matrix of initial prices of assets over all time intervals, $P=\left(P_{i j}\right)$ the $N \times N$ matrix of portfolios (rows) over time where $P_{i j}=x_{0, i j} w_{i j}$. Using the above notation, the expected stock portfolio return vector and risk matrix are then given respectively by

$$
\bar{R}=\sum_{j=1}^{N} w_{j} \mu_{j} \quad \text { and } \quad r=\sum_{i=1}^{N} \sum_{j=1}^{N}\left(w_{i}, w_{j}\right) \sigma_{i j}
$$

where

$$
\mu_{j}=\frac{1}{N} \sum_{i=j}^{n} P_{j}
$$

is the arithmetic mean of column $j$ of the portfolio matrix, $\left(w_{i}, w_{j}\right)$ is the scalar product of $w_{i}$ and $w_{j}$.

We also define a risk aversion parameter $\lambda \in[0,1]$ to present what is known as the weighted formulation of the portfolio optimization problem. Observation of $\lambda$ reveals that when it is close to zero, the weights shift toward stocks yielding high returns and when close to one, the weights shift toward combinations of stocks yielding low volatility in the efficient set. Define the cost function

$$
f=\lambda \sum_{i=1}^{N} \sum_{j=1}^{N}\left(w_{i}, w_{j}\right) \sigma_{i j}-(1-\lambda) \sum_{j=1}^{N} w_{j} \mu_{j}
$$

Now let us formulate our modified problem

a) Find

$$
\min _{w_{i}, w_{j}} f
$$

\section{b) Subject to:}

i) $\sum_{j=1}^{N} w_{i j}=1$ for all $i=1, \cdots, N$ (Basic constraint)

ii) $v_{j} \leq w_{i j} \leq \delta_{j}, 0 \leq v_{j}<\delta_{j} \leq 1$ for all $i, j$ (Boundary constraint)

iii) $\operatorname{Pr}\left(\ell_{P(t)} \leq 0.01\right) \leq 0.01$ (Probability constraint)

iv) $\sum_{i=1}^{N} Z_{i j} \leq K_{j}$ for all $j$ where $Z_{i j}=\left\{\begin{array}{l}1 \text {; if } w_{i j}>v_{j} \\ 0 \text {; otherwise }\end{array}\right.$ (Cardinality constraint)

$\ell_{P(t)}$ is the loss in portfolio $\mathrm{P}$ at time $\mathrm{t}$ and condition (i) requires that each row of the weight matrix sums to 1 , with condition (ii) requiring two vectors $v=\left(v_{j}\right), \delta=\left(\delta_{j}\right)$ consisting of (possibly distinct) boundaries. Condition (iii) stipulates that the loss of any new portfolio created from an old one should be less than $1 \%$ to be acceptable. In this paper we will not go into detail on the GA implementation, rather focusing on the analysis of its outputs. For the interested 
reader, the algorithm may be found in [1] [18] [19] and [20]. The next section will present our results and the comparative analysis of various scenarios of investment.

\section{Results and Comparative Analysis}

We apply our method to the historical data of eight years worth of assets (Table 1). Those assets are widely used indexes: CAC 40, FTSE 100, S \& P 500, Wilshire 5000, NASDAQ, Barclays 7 - 10 Year Treasury (IEF), MSCI EAFE Index Fund, and Gold.

In the mathematical interpretation, this data is an 8 by 8 matrix where columns represent assets and rows are the values of the asset. The values are normalised beforehand for stability reasons. Fixing a given year (row), then by multiplying each asset by the corresponding weight of the above matrix data in and summing the results, we obtain the portfolio value at the given year (row). Below we give the results obtained for the values $K=3,4,7,8$.

Comments in Figures 1-4 show the results obtained with three experiments (that is, three different initial weight matrices were generated at random, subject to the given conditions) on which the GA was run five times for each of the four different values of K. Each table exhibits the following: Best cost value attained; Gain from minimally reliable portfolio or row; Mean and standard deviation (SD) of each experiment, Total \% Gain in Matrix portfolio value from a randomly produced initial weight matrix $w_{0}$; Maximum Mean Portfolio Value in Best fit matrix over all generations, and Minimum Standard deviation of Portfolio Value in the best fit matrix over all generations obtained by the GA. It can be seen that when the investor decides on diversification in the portfolio constructed with $K=3$ and $K=7$ assets, the expected portfolio value is higher than in the portfolio constructed with $K=4$ and $K=8$ assets.

\section{Discussion}

The proposed comparative analysis shows that high return on a portfolio may be

Table 1. The initial prices used in our experiments. Each entry gives the value of the applicable asset on the date as close as possible to $1^{\text {st }}$ January in the given year.

\begin{tabular}{ccccccccc}
\hline Year & CAC 40 (Close) & $\begin{array}{c}\text { FTSE 100 } \\
\text { (Close) }\end{array}$ & $\begin{array}{c}\text { S \& P 500 } \\
\text { (Close) }\end{array}$ & $\begin{array}{c}\text { DJW 5000 } \\
\text { (Close) }\end{array}$ & $\begin{array}{c}\text { NASDAQ Comp. } \\
\text { (Close) }\end{array}$ & $\begin{array}{c}\text { BARCLAY S 7610 } \\
\text { vr treas (Close) }\end{array}$ & $\begin{array}{c}\text { MCSI EAFE } \\
\text { Close }\end{array}$ & $\begin{array}{c}\text { Gold London } \\
\text { PM Fix }\end{array}$ \\
\hline 2003 & 2937.88 & 3567.40 & 855.70 & 8125.10 & 1320.91 & 85.49 & 94.75 & 343.80 \\
2004 & 3638.44 & 4390.70 & 1131.13 & $11,029.20$ & 2066.15 & 85.56 & 138.32 & 416.25 \\
2005 & 3913.69 & 4852.30 & 1181.27 & $11,642.60$ & 2962.41 & 85.69 & 157.20 & 427.75 \\
2006 & 4947.99 & 5760.30 & 1280.08 & $12,953.60$ & 2305.82 & 83.32 & 62.86 & 530.00 \\
2007 & 5608.31 & 6203.10 & 1438.24 & $14,489.70$ & 2463.93 & 82.11 & 74.24 & 639.75 \\
2008 & 4869.79 & 5879.80 & 1378.55 & $13,896.70$ & 2389.86 & 89.93 & 72.34 & 846.75 \\
2009 & 2973.92 & 4149.60 & 825.88 & 8335.60 & 1476.42 & 94.70 & 38.70 & 874.50 \\
2010 & 3739.46 & 5188.50 & 1073.87 & $11,099.40$ & 2147.35 & 90.70 & 52.48 & 1121.50 \\
\hline
\end{tabular}


Best Cost Value Attained

$\begin{array}{lrrr}\text { Experiment } & 1 & 2 & 3 \\ & -4.0576 & -3.1532 & -3.2158 \\ & -3.7509 & -3.5084 & -3.2579 \\ & -3.7239 & -3.5287 & -3.3074 \\ & -3.9396 & -3.6883 & -3.6450 \\ & -3.6572 & -3.5652 & -3.4650 \\ \text { Mean } & -3.8258 & -3.4888 & -3.3782 \\ \text { SD } & 0.1667 & 0.2002 & 0.1765\end{array}$

Total \% Gain Matrix Portfolio Value from $\mathrm{Na}$ Experiment 161.798176 .1333107 .9646 138.3214 99.3944 111.6863 135.897999 .0104114 .6000 151.4145112 .8415140 .3199 130.4457101 .8255137 .4615 Mean $\quad 143.5755 \quad 97.841120 .4065$ $\begin{array}{llll}\text { SD } & 12.7716 & 13.3781 & 13.3311\end{array}$

Minimal Reliability

$\begin{array}{lccrr}\text { Experiment } & 1 & 2 & 3 \\ & 0.8216 & 0.0026 & 0.2423 \\ & 0.3054 & 0.0320 & 0.5216 \\ & 0.2200 & 0.1018 & 0.1766 \\ & 0.4205 & 0.2711 & 0.1971 \\ \text { Mean } & 0.3395 & 0.2730 & 0.4498 \\ \text { SD } & 0.4214 & 0.1361 & 0.3175 \\ & 0.2350 & 0.1292 & 0.1575\end{array}$

ES: Expected Shortfall

SD: Standard Deviation

Figure 1. Case studied $K=8$.

Best Cost Value Attained

$\begin{array}{lrrr}\text { Experiment } & 1 & 2 & 3 \\ & -3.9880 & -3.4632 & -3.8981 \\ & -3.9390 & -3.6053 & -3.6396 \\ & -3.4084 & -3.2892 & -3.1831 \\ & -4.2875 & -3.3005 & -3.0790 \\ \text { Mean } & -3.8009 & -3.4567 & -3.1234 \\ \text { SD } & -3.8846 & -3.4230 & -3.3846 \\ & 0.3200 & 0.1312 & 0.3643\end{array}$

$\%$ Gain from Mininally Reliable Row of $\mathrm{w}_{0}$

$\begin{array}{lrrr}\text { Experiment } & 1 & 2 & 3 \\ & 271.2737 & 77.7111 & 62.0644 \\ & 163.8190127 .8926 & 277.1561 \\ & 215.3108 & 79.5455 & 95.8385 \\ & 175.5889 & 16.6523 & 183.2692 \\ & 166.2527 & 37.8971 & 257.3276 \\ \text { Mean } & 198.4490 & 67.9397 & 175.1312 \\ \text { SD } & 45.6787 & 42.8879 & 95.2667\end{array}$

Best ES Attained

Experiment $1 \quad 2 \quad 2 \quad 3$

0.56180 .42020 .4273 $\begin{array}{lll}0.5122 & 0.4746 & 0.4356\end{array}$ 0.50720 .47410 .4414 $0.5390 \quad 0.50800 .4943$ 0.49540 .48100 .4666

Mean $\quad 0.52310 .47160 .4530$ SD $\quad 0.0269 \quad 0.03190 .0274$

Maximum Mean Portfolio Value in Best Fit Matrix over all Generations

$\begin{array}{lrrr}\text { Experiment } & 1 & 2 & 3 \\ & 0.5629 & 0.4206 & 0.4283 \\ & 0.5125 & 0.4762 & 0.4359 \\ & 0.5072 & 0.4753 & 0.4419 \\ & 0.5406 & 0.5083 & 0.4949 \\ & 0.4955 & 0.482 & 0.4684 \\ \text { Mean } & 0.5238 & 0.4725 & 0.4539 \\ \text { SD } & 0.0275 & 0.0319 & 0.0275\end{array}$

Min SD Portfolio Value in Best Fit Matrix over all Generations

$\begin{array}{lccr}\text { Experiment } & 1 & 2 & 3 \\ & 0.0620 & 0.1001 & 0.0475 \\ & 0.0707 & 0.0910 & 0.0696 \\ & 0.0793 & 0.1175 & 0.0731 \\ & 0.0397 & 0.0586 & 0.0475 \\ \text { Mean } & 0.0451 & 0.0478 & 0.0365 \\ \text { SD } & 0.0594 & 0.0830 & 0.0548 \\ & 0.0168 & 0.0291 & 0.0158\end{array}$

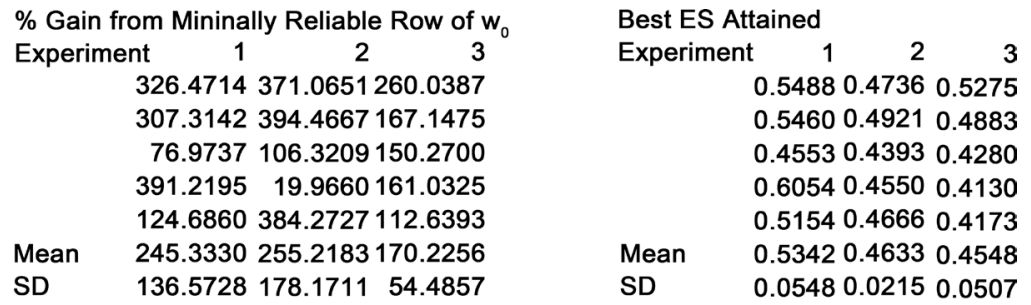

Total \% Gain Matrix Portfolio Value over Generations Maximum Mean Portfolio Value in Best Fit Matrix over all Generations

$\begin{array}{lrrrrrrr}\text { Experiment } & 1 & 2 & 3 & \text { Experiment } & 1 & 2 & 3 \\ & 138.4712 & 99.5876155 .8112 & & 0.5498 & 0.4740 & 0.5285 \\ & 136.8894107 .7098136 .3791 & & 0.5461 & 0.4933 & 0.4883 \\ & 99.6265 & 85.3261107 .7539 & & 0.4602 & 0.4402 & 0.4292 \\ & 163.7086 & 87.6660100 .3551 & & 0.6079 & 0.4457 & 0.4139 \\ & 123.8110 & 97.0608102 .2400 & & 0.5160 & 0.4680 & 0.4178 \\ \text { Mean } & 132.5027 & 95.4701120 .5078 & \text { Mean } & 0.5360 & 0.4643 & 0.4556 \\ \text { SD } & 23.3717 & 9.1255 & 24.4986 & \text { SD } & 0.0539 & 0.0217 & 0.0506\end{array}$

Minimal Reliability

\begin{tabular}{lrrr} 
Experiment & 1 & 2 & 3 \\
& 0.5095 & 0.5308 & 0.2405 \\
& 0.1375 & 0.0761 & 0.3261 \\
& 0.0314 & 0.0914 & 0.0909 \\
& 0.0648 & 0.2464 & 0.0320 \\
Mean & 0.1460 & 0.1398 & 0.0093 \\
SD & 0.1778 & 0.2169 & 0.1398 \\
\hline
\end{tabular}

Min SD Portfolio Value in Best Fit Matrix over all Generation

\begin{tabular}{lrrr} 
Experiment & 1 & 2 & 3 \\
& 0.0418 & 0.0828 & 0.0458 \\
& 0.0664 & 0.0793 & 0.0672 \\
& 0.0352 & 0.0556 & 0.0700 \\
& 0.0476 & 0.0792 & 0.0772 \\
Mean & 0.0806 & 0.0756 & 0.0909 \\
SD & 0.0543 & 0.0745 & 0.0702 \\
\hline & 0.0187 & 0.0109 & 0.0165
\end{tabular}

ES: Expected Shortfall

SD: Standard Deviation

Figure 2. Case studied $K=7$. 
Table 2. This table shows summary statistics of overall results, that is those of cases studied ( $K=3,4,7$ and 8 ). Shown for each value of $K$ are, respectively, the means of the quantities shown on this table. In the case of the total percentage gain in portfolio value in the whole matrix, this is simply the gain from following the portfolio found at each time step.

\begin{tabular}{ccccc}
\hline K & 3 & 4 & 7 & 8 \\
\hline Best Cost & -3.408 & -3.467 & -3.564 & -3.564 \\
Max (Normalized) Mean PV & 0.485 & 0.478 & 0.485 & 0.483 \\
Min (Normalized) SD PV & 0.208 & 0.193 & 0.066 & 0.066 \\
Min Rel & 0.216 & 0.211 & 0.178 & 0.292 \\
Min Rel \% Gain & 156.114 & 51.143 & 223.592 & 147.173 \\
Total \% Gain & 86.517 & 87.052 & 116.160 & 120.608 \\
Best ES & 0.485 & 0.478 & 0.484 & 0.483 \\
\hline
\end{tabular}

achieved with both a relatively low $(K=3)$ and a relatively high $(K=7)$ number of assets and differentiated risks (which have, respectively, a relatively high and low risk). We recall that we choose to measure the risk of a portfolio by the normalised standard deviation in its value over the given number of trials. We observed that the "mean" portfolio for $K=3$ has a high mean portfolio value with high risk, and an investor who hopes to increase his or her portfolio value in an aggressive manner may chose such an allocation procedure. This approach will then challenge the Markowitz belief that the only optimal portfolio is the one with higher expected mean portfolio value and smaller risk or standard deviation. But generally speaking, most nonaggressive investors will tend to follow the Markowitz theory when constructing an investment strategy.

Our results have again highlighted the possibility of constructing an optimal portfolio with both low or relatively high risk (which is almost twice the lowest risk; see Table 2). The advantage of our approach is to show both possibilities which gives more choice to the investor, but the main disadvantage is that our number of total assets $(N)$ is not very large (eight assets) and the difference between the number of assets which are invested is also relatively small (for $K=3$ and $K=7$ out of $N=8$, the numbers are relatively close, numerically speaking, but in the problem they produced interesting and distinct results that could be used in practice to understand and analyse portfolio selection in a comprehensive manner).

\section{Conclusions}

In this paper a comparative study of the Constrained Portfolio Optimization Problem with cardinality constraint is investigated. The experimental studies have been undertaken on widely used indexes form the Period of January 2003 to January 2010. Our conclusions are as follows:

1) The diversification in asset numbers less than the total number of assets (that is, for $K<N$ ) may increase the expected portfolio return with different 
reliabilities. We found that the "mean" portfolios for $K=3$ and $K=7$ both had high mean portfolio values, but the $K=7$ portfolio had the best reliability, meaning that the investor will choose either $K=3$ or $K=7$ depending on how reliable it is likely to be.

2) Important information such as the gain from minimally reliable portfolio or row, mean and standard deviation (SD) of each experiment, total percentage gain in matrix portfolio value, and the maximum mean and minimum standard deviation in portfolio value may play an important role in optimal portfolio selection and management.

3) The "mean" portfolio for $K=7$ has the highest "most reliable row" gain percentage, indicating that the preference of investors may be higher on such a portfolio compared to that of $K=3$, even though they have identical mean portfolio values.

Future directions of this research include: investigating new concepts for diversification in large portfolios and comparing with results of diversification in small portfolios, therefore building a theory that could link those two approaches.

\section{Acknowledgements}

We are grateful to all colleagues for discussions and suggestions which helped us to improve the presentation of this paper. We thank the Waseda University and University Paris 1 , for the generous research support during the preparation of this work. This work was also supported by the Grant-in-Aid for Scientific Research, both of which are part of the MEXT (Ministry of Education, Culture and Sports, Sciences and Technology of Japan). We finally thank Professor Yuichi Sakumura for useful comments and the SAKURA Research \& Consulting for bringing up such an interesting research topic.

\section{References}

[1] Mulvey, J.M. (1993) Incorporating Transaction Costs in Models for Asset Allocations, Financial Optimization, Stavros A. Zenios. Cambridge University Press, Cambridge.

[2] Tadonki, C. and Vial, J.Ph. (2014) Portfolio Selection with Cardinality and Bound Constraints. http://www.omegacomputer.com/staff/tadonki/PaperForWeb/Tadonki_PF.pdf

[3] Dietmar, M. (2005) Portfolio Management with Heuristic Optimization. Springer-Verlag, New York.

[4] Goldberg, D.E. (1989) Genetic Algorithms in Search, Optimization and Machine Learning. Addison-Wesley, Boston.

[5] Jimbo, H.C. and Craven, M.J. (2011) Optimal Stock Investment with Stochastic Constraints. Proceedings of NACA (Nonlinear Analysis and Convex Analysis), Busan, Korea, 2-5 August 2011, Busan.

[6] Berger, A.J., Glover, F. and Mulvey, J.M. (1995) Solving Global Optimization Problems in Long-Term Financial Planning, Statistics and Operations Research, Technical Report. Princeton University, Princeton, NJ. 
[7] Chan, M.C., Wong, C.C., Cheung, B. and Tang, G. (2005) Advance in Soft Computing. Vol. 29, Springer, Berlin, Heidelberg.

[8] Chan, M.C., Wong, C.C., Cheung, B. and Tang, G. (2005) Advance in Soft Computing. Vol. 29, Springer, Berlin, Heidelberg.

[9] Dentcheva, D. and Ruszczynski, A. (2003) Optimization with Stochastic Dominance Constraints. SIAM Journal on Optimization, 14, 548-566. https://doi.org/10.1137/S1052623402420528

[10] Elton, E.J., Gruber, M.J., Brown, S.J. and Goetzmann, W.N. (2003) Modern Portfolio Theory and Investment Analysis. 6th Edition, John Wiley \& Sons, Hoboken, NJ.

[11] Mansini, R. and Speranza, M.G. (1999) Heuristic Algorithms for the Portfolio Selection Problem with Minimum Transaction Lots. European Journal of Operational Research, 114, 219-223. https://doi.org/10.1016/S0377-2217(98)00252-5

[12] Shetty, B., Mulvey, J.M. and Rosenbaum, D.P. (1997) Strategic Financial Risk Management and Operations Research. European Journal of Operational Research, 97, 116.

[13] Duan, Y.C. (2007) A Multi-Objective Approach to Portfolio Optimization. http://www.rose-hulman.edu/mathjournal/archives/2007/vol8-n1/paper12/v8n1-12 pd.pdf

[14] Elton, E.J. (1995) Portfolio Theory and Investment Analysis. Wiley, Hoboken.

[15] Jimbo, H.C. and Craven, M.J. (2011) Unconstrained Optimization in a Stochastic Cellular Automata System. Journal of Nonlinear Analysis and Optimization, 1, 103-110.

[16] Jimbo, H., Ouentcheu, A. and Bozeman, R.E. (2004) Portfolio Optimization with the Growth Model. Journal of Nonlinear and Convex Analysis, 131-141.

[17] Pearson, N. (2002) Risk Budgeting: Portfolio Problem Solving with Value-at-Risk, John Wiley \& Sons, Hoboken.

[18] Osyczka, A. (2002) Evolutionary Algorithms for Single and Multicriteria Design Optimization. Physica-Verlag, Heidelberg.

[19] Pappa, G., Pavkaa, S.Z., Novakc, M.A. and Kondora, I. (2005) Random Matrix Filtering in Portfolio Optimization. Acta Physica Polonica B, 36, 2757-2765.

[20] Winker, P. (2001) Optimization Heuristic in Economic Application of Threshold Accepting. Wiley, Hoboken.

\section{Scientific Research Publishing}

Submit or recommend next manuscript to SCIRP and we will provide best service for you:

Accepting pre-submission inquiries through Email, Facebook, LinkedIn, Twitter, etc. A wide selection of journals (inclusive of 9 subjects, more than 200 journals)

Providing 24-hour high-quality service

User-friendly online submission system

Fair and swift peer-review system

Efficient typesetting and proofreading procedure

Display of the result of downloads and visits, as well as the number of cited articles

Maximum dissemination of your research work

Submit your manuscript at: http://papersubmission.scirp.org/

Or contact ojs@scirp.org 